\title{
UTP is a regulator of in vitro and in vivo angiogenic properties of cardiac adipose-derived stem cells
}

\author{
Marion Vanorlé ${ }^{1} \cdot$ Anne Lemaire $^{1} \cdot$ Larissa di Pietrantonio $^{1} \cdot$ Michael Horckmans $^{1} \cdot$ Didier Communi $^{1}$ (I)
}

Received: 29 March 2021 / Accepted: 11 July 2021

(c) The Author(s), under exclusive licence to Springer Nature B.V. 2021

\begin{abstract}
The ability of cardiac adipose-derived stem cells (cADSC) to differentiate into multiple cell types has opened new perspectives in cardiac cell-based regenerative therapies. P2Y nucleotide receptors have already been described as regulators of adipogenic differentiation of cADSC and bone marrow-derived stem cells. In this study, we defined UTP as a regulator of cADSC endothelial differentiation. A daily UTP stimulation of cADSC during endothelial predifferentiation increased their capacity to form an endothelial network in matrigel. Additionally, pro-angiogenic UTP target genes such as epiregulin and hyaluronan synthase-1 were identified in predifferentiated cADSC by RNA sequencing experiments. Their regulation by UTP was confirmed by qPCR and ELISA experiments. We then evaluated the capacity of UTP-treated predifferentiated cADSC to increase post-ischemic revascularization in mice subjected to left anterior descending artery ligation. Predifferentiated cADSC treated or not with UTP were injected in the periphery of the infarcted zone, 3 days after ligation. We observed a significant increase of capillary density 14 and 30 days after UTP-treated predifferentiated cADSC injection, correlated with a reduction of cardiac fibrosis. This revascularization increase was not observed after injection of UTP-treated cADSC deficient for UTP and ATP nucleotide receptor $\mathrm{P}_{2} \mathrm{Y}_{2}$. The present study highlights the $\mathrm{P}_{2} \mathrm{Y}_{2}$ receptor as a regulator of cADSC endothelial differentiation and as a potential target for the therapeutic use of cADSC in post-ischemic heart revascularization.
\end{abstract}

Keywords Adipose stem cells $\cdot$ Cardiac $\cdot$ Angiogenesis $\cdot$ Ischemia $\cdot$ Extracellular nucleotides $\cdot$ P2Y receptors

\section{Introduction}

Revascularization and restoration of blood flow remain the mainstay of the current therapeutic interventions to myocardial ischemia. In recent years, stem cell transplantation has been recognized as a promising strategy of therapeutic revascularization developed for patients with myocardial infarction (MI) [1]. Among adult stem cell sources, adipose-derived stem cells (ADSC) have emerged as the ideal cell source for therapeutic approaches due to their ease of acquisition, high yields, proliferative growth, multidifferentiation capacity, and low immunogenicity [2]. ADSC are

Michael Horckmans and Didier Communi contributed equally to this work

Didier Communi

Didier.Communi@ulb.be

1 Institute of Interdisciplinary Research, IRIBHM, Université Libre de Bruxelles, ULB, Building C (5th floor), Campus Erasme, 808 Route de Lennik, 1070 Brussels, Belgium known for producing pro-angiogenic, anti-apoptotic, and anti-inflammatory cytokines and growth factor secretions in implanted myocardium after ischemic injury [3, 4]. Undeniably, plenty of basic research and clinical trials have clearly demonstrated that ADSC can enhance heart function, reducing myocardial infarction and promoting vascularization of MI hearts [5, 6].

A growing body of evidence demonstrated that the therapeutic proangiogenic effects of ADSC are attributed more to their secretory potential than to their cardiac differentiation capacity and directly integrating damaged tissue [7]. Based on this new insight, the current research directions encompass efforts to elucidate, increase, and regulate the paracrine mechanisms underlying tissue repair and regeneration with stem or progenitor cell transplantation. The pretreatment of ADSC before transplantation with biochemical or physical factors might be one of the most encouraging strategies to improve ADSC cardioprotective and proangiogenic therapeutic effects. Indeed, hypoxic preconditioning of cell culture enhances ADSC proliferation and upregulates the transcriptional activation of proangiogenic factors in the 
ischemic site [8]. Moreover, melatonin pretreatment has been shown to promote the survival of transplanted ADSC and stimulate their paracrine potential in a rat MI model [9]. Nevertheless, a lot of progress still needs to be made to optimize the responsiveness and the angiogenic capabilities of transplanted-ADSC in myocardial infarction.

Cardiac adipose-derived stem cells (cADSC), isolated from C57BL/6 mice, have a high potential to differentiate into cardiovascular lineage cells (i.e., cardiomyocytes, endothelial cells, and vascular smooth muscle cells) in vitro compared with stem cells derived from other fat depots such as subcutaneous, visceral, and subscapular adipose tissues. These recent findings might give rise to a new autologous stem cell therapy for patients with myocardial infarction [10]. Myocardial ischemia is associated with an important release of extracellular nucleotides by cardiomyocytes and cardiac endothelial cells [11]. These extracellular nucleotides are potent mediators of physiologic and pathologic functions contributing to the inflammatory and fibrotic response within the injured myocardium. The $\mathrm{P}_{2} \mathrm{Y}_{2}$ receptor, ubiquitously expressed throughout the body, is an UTP/ATP G-protein-linked nucleotide receptor coupled to the phosphoinositide signaling pathway [12]. Previous data have demonstrated that $\mathrm{P} 2 \mathrm{Y}_{2}$ subtype activation can significantly reduce cardiomyocyte death induced by hypoxia and that UTP administration to rats gives rise to a reduced infarct size and superior myocardial function [13]. The $\mathrm{P}_{2} \mathrm{Y}_{4}$ receptor, another UTP G-protein-linked nucleotide receptor [14], is also involved in cardioprotection but through very distinct mechanisms and effects compared to the $\mathrm{P} 2 \mathrm{Y}_{2}$ receptor. Our laboratory demonstrated that the loss of $\mathrm{P}_{2} \mathrm{Y}_{4}$ receptor is associated with a protection against infarction and a decrease in cardiac inflammation, fibrosis, and permeability [15].

In the last years, purinergic receptors have been reported as key elements regulating proliferation, differentiation, cell death, and successful engraftment of stem cells from diverse origins [16, 17]. Zippel et al. proposed for the first time that the combination of several $\mathrm{P} 2$ receptors plays a role in the adipogenic and osteogenic differentiation of adult stem cells. The $\mathrm{P}_{2} \mathrm{Y}_{2}$ receptor seems to be specific for osteogenic differentiation while the $\mathrm{P}_{2} \mathrm{Y}_{4}$ receptor, also activated by UTP ligand, seems to be a pivotal regulator in both adipogenic and osteogenic differentiation processes [18]. More recently, we demonstrated the respective negative and positive effects of the $\mathrm{P}_{2} \mathrm{Y}_{4}$ and $\mathrm{P} 2 \mathrm{Y}_{2}$ receptors on adipogenic differentiation of mouse cADSC [19, 20]. Thereby, therapeutic applications based on the regulation of purinergic-mediated cell differentiation signaling may provide novel mechanisms for cell therapy and endogenous tissue repair.

In the present study, we investigated if the ligand UTP was a regulator of in vitro endothelial differentiation process of cADSC. Moreover, we evaluated the proangiogenic therapeutic effects of UTP-pretreated cADSC in a murine in vivo model of myocardial infarction.

\section{Methods}

\section{Animals}

Eight- to twelve-week-old $\mathrm{P}_{2} \mathrm{Y}_{2}$ receptor-null CD1/C57B16 female and male mice were generated and kindly gifted by Dr. B. Koller [21].

\section{CADSC isolation and endothelial predifferentiation}

cADSC were isolated from the stromal vascular fraction of mouse adipose tissue and their purity was checked as previously described [19, 20,22]. The cardiac adipose tissue, situated between the visceral pericardium and the epicardium (including epi- and pericardic fat depots), was freshly harvested. Adipose tissue was washed in phosphate-buffered saline (PBS) and minced, followed by a digestion in collagenase $\mathrm{A}(2.5 \mathrm{~g} / \mathrm{L})$ at $37^{\circ} \mathrm{C}$ for $45 \mathrm{~min}$. The digested tissue was filtered through a nylon mesh $(500 \mathrm{~nm})$ and centrifuged at $500 \mathrm{~g}$ for $5 \mathrm{~min}$. The supernatant containing adipocytes and debris was discarded. A red blood cell lysis was performed on the pelleted cells by an osmotic shock. After the last centrifugation at $500 \mathrm{~g}$ for $5 \mathrm{~min}$, the resultant pellet, named the stromal vascular fraction, was suspended in endothelial growth medium-2, (EGM-2; Lonza) with SingleQuots containing 5\% fetal bovine serum, acid ascorbic, epidermal growth factor, hydrocortisone, bovine brain extract, and gentamycin/amphotericin-B, at a density of $5 \times 10^{4}$ per $\mathrm{cm}^{2}$ under $5 \% \mathrm{O}_{2}$ and $37{ }^{\circ} \mathrm{C}$ conditions for 7 days. cADSC were selected by their high plastic adhesion. Non-adherent cells were removed after $24 \mathrm{~h}$ and the culture medium was subsequently replaced every 2 days. During the endothelial differentiation, the cADSC cultures were unstimulated or stimulated daily with UTP $100 \mu \mathrm{M}$.

\section{In vitro matrigel assays}

$\mu$-Slide 15-Well Angiogenesis (Ibidi, GmbH, Martinsried, Germany) was covered with $20 \mu \mathrm{L}$ of Matrigel $®$ Basement Membrane Matrix, Growth Factor Reduced, Phenol RedFree (Corning). cADSC, cultured during 7 days in EGM2 and stimulated daily with or without UTP $100 \mu \mathrm{M}$, were seeded at a density of $2 \times 10^{4} / 45 \mu \mathrm{L}$ in EGM 2 medium. The plates were incubated at $37{ }^{\circ} \mathrm{C}$ in a $5 \% \mathrm{CO}_{2}$ for up to $20 \mathrm{~h}$. Pictures were then taken with an inverted-phase-contrast light microscope Zeiss at a magnification of $\times 25$. The total capillary tube length, loop area, and loop number, three specific angiogenic measurements, were quantified $20 \mathrm{~h}$ after 
seeding by using the automated software Wimasis WimTube (Wimasis GmbH, Munich, Germany).

\section{Myocardial infarction model}

Wild-type mice were anesthetized with a mixture of midazolam $(5 \mathrm{mg} / \mathrm{kg})$, medetomidine $(0.5 \mathrm{mg} / \mathrm{kg})$, and fentanyl $(0.05 \mathrm{mg} / \mathrm{kg})$, intubated and mechanically ventilated (rate 130 stroke/min, tidal volume $0.13 \mathrm{~mL}$ ). An optical magnification loop was used for better visualization of the operation field. A left-side thoracotomy was performed between the $3^{\text {rd }}$ and the $4^{\text {th }}$ rib, and the pericardium was incised. Once the heart was exposed, MI was induced by the permanent ligation of the left anterior descending artery (LAD) proximal to its bifurcation from the main stem. Successful performance of coronary occlusion was verified by blanching of the myocardium distal to the coronary ligation. The thoracic incision was then closed with a $6-0$ silk suture, at the muscle tissue and the skin. An antagonist cocktail of naloxone $(1.2 \mathrm{mg} /$ $\mathrm{kg})$, flumazenil $(0.5 \mathrm{mg} / \mathrm{kg})$, and atipamezole $(2.5 \mathrm{mg} / \mathrm{kg})$ was finally administered to the mice to reverse the general anesthesia.

\section{Quantification of injected EdU-labeled predifferentiated CADSC in ischemic heart}

Wild-type mice were injected with predifferentiated cADSC pre-labeled with EdU $(10 \mu \mathrm{M})$ in 3 days post-MI hearts $\left(3 \times 10^{5}\right.$ cells/heart). ADSC predifferentiated in EGM2 for 7 days and unstimulated or stimulated daily with UTP $100 \mu \mathrm{M}$ were detached with trypsin/EDTA and resuspended in PBS to be counted. Then the cells were injected at 3 different sites along the infarct border zone with a final volume of $10 \mu \mathrm{L}$ at each site $\left(3 \times 10^{5}\right.$ cells/ heart). Two hours or 4 days after injection, hearts were collected, washed in cold PBS, dried and frozen directly in Tissue-Tek OCT compound (VWR Scientific), and frozen at $-80^{\circ} \mathrm{C}$. The EdU staining was revealed with the Click-iT ${ }^{\mathrm{TM}}$ EdU Alexa Fluor ${ }^{\mathrm{TM}} 488$ Imaging Kit (Thermo Fisher Scientific, Waltham, MA).

\section{Quantification of revascularization after CADSC injection in mouse ischemic heart}

Three days after LAD, mice underwent a second thoracotomy as described above. This was followed by intramyocardial cADSC injections. Wild-type or $\mathrm{P}_{2} \mathrm{Y}_{2} \mathrm{KO}$-unlabeled predifferentiated cADSC unstimulated or stimulated daily with UTP $100 \mu \mathrm{M}$ were injected at 3 different sites along the infarct border zone with a final volume of $10 \mu \mathrm{L}$ at each site $\left(3 \times 10^{5}\right.$ cells/heart). Fourteen days or 30 days after injection, hearts were collected, washed in cold PBS, dried and embedded directly in Tissue-Tek OCT compound (VWR Scientific), and frozen at $-80^{\circ} \mathrm{C}$.
The vascularity of the ischemic myocardium was assessed by two specific stains recognizing the presence of capillaries and arterioles. In brief, 8 - $\mu \mathrm{m}$-thick heart cryosections were stained with antibodies against biotinylated Isolectin B4 (IB-4) Alexa Fluor TM 647-conjugated (Invitrogen, 1/200 ON $4{ }^{\circ} \mathrm{C}$ ) and $\alpha$-smooth muscle cell actin ( $\alpha$-SMA) Cy $3{ }^{\mathrm{TM}}$ conjugated (Sigma-Aldrich, 1/200 $1 \mathrm{~h}$ at RT). Densities of the capillary (IB-4 staining) and arteriole (IB- $4+\alpha$-SMA staining) were quantified in the peri-infarct myocardium. The final data were expressed as the number of arterioles ( $<20 \mu \mathrm{m}$ in diameter) or capillaries per square millimeter; the percentage is referring to the ratio between the control condition (PBS injection) and the cADSC injection condition. The area of the infarct border zone was determined as the $0.5-\mathrm{mm}$ region of histologically intact myocardium surrounding the evident infarct-related fibrocellular region. These analyses were performed by using the Image J software by examining around 20 or 60 fields for arteriole and capillary density respectively, per heart at $\times 100$ magnification.

\section{Quantification of fibrosis area in ischemic hearts}

Thirty days after injection of untreated or UTP-treated cADSC, heart sections of ischemic mice were cut, fixed in Bouin's solution, and stained with Masson's trichrome. The fibrosis was quantified as the relative surface of the blue staining on five cryosections $(8 \mu \mathrm{m})$ of the ischemic heart using the ImageJ software and normalized to the left ventricle area.

\section{RNA-sequencing experiments}

RNA-sequencing experiments were realized on cADSC cultured in EGM2 for 7 days, unstimulated or with daily stimulation with UTP $100 \mu \mathrm{M}$. cADSC were harvested and RNA was extracted using TRIzol reagents (Thermo Fisher Scientific, Waltham, MA) and cleaned on the RNeasy kit column (Qiagen). In total, $1 \mu \mathrm{g} / 50 \mu \mathrm{L}$ of RNA was engaged and the quality was checked using a Bioanalyzer 2100 (Agilent technologies). cDNA libraries were obtained using the TruSeq Stranded mRNA Library Prep kit (NuGen) following the manufacturer's recommendations. The multiplex libraries $(18 \mathrm{pM})$ were loaded on flow cells and sequences were produced using a HiSeq PE Cluster Kit v4 and TruSeq SBS Kit v3-HS from a Hiseq 1500 (Illumina). Approximately, 25 million of paired-end reads per sample were mapped against the mouse reference genome (GRCm38.p4/mm10) using the STAR software to generate read alignments for each sample. Annotations Musmusculus GRC38.87.gtf were obtained from ftp.Ensembl.org. After transcripts assembling, gene-level counts were obtained using HTSeq. Genes with FPKM $\geq 2$ in cADSC + UTP RNA and with FPKM 
$\mathrm{cADSC}+\mathrm{UTP} / \mathrm{FPKM} \mathrm{cADSC}+\mathrm{UTP} \geq 2$ were selected and sorted in the function of pathways.

\section{Quantitative real-time qPCR}

Total mRNA was extracted using TRIzol reagents (Thermo Fisher Scientific, Waltham, MA) and an RNeasy kit column (Qiagen, Hilden, Germany) from cADSC cultured in EGM2 for 7 days, with daily UTP stimulation or unstimulated. mRNA was reverse transcribed with random hexamers and Superscript ${ }^{\circledR}$ II Reverse Transcriptase (Invitrogen, Life Technologies). Gene expression was then analyzed by quantitative PCR-based amplification on 8 ng cDNA using specific primers and Kapa SYBR ${ }^{\circledR}$ Fast Rox TM Low (Roche, Basel, Switzerland). Reactions were run on a 7500 Fast Time PCR System (Applied Biosystems, Foster City, CA, USA). The expression of RPL32 was employed as a housekeeping gene to normalize the expression levels and the results were expressed on $2^{\wedge-\Delta \mathrm{CT}}$.

\section{Quantification of epiregulin and hyaluronan synthase 1 levels in CADSC supernatants by ELISA}

Supernatants of cADSC cultured in EGM2 for 7 days were collected and total proteins were quantified using Pierce TM BCA Protein Assay Kit (Thermo Fisher Scientific). Epiregulin (Thermo Fisher Scientific) and hyaluronan synthase 1 (MyBioSource) levels were measured by enzyme-linked immunosorbent assay (ELISA), according to the manufacturer's procedure.

\section{Statistical analysis}

Data were expressed as mean \pm standard error of the mean (SEM) for in vitro and in vivo studies. Endpoint comparisons with two groups were performed using an unpaired two-tailed Student's $t$-test and Mann-Whitney test for parametric and nonparametric data, respectively (Prism Software, version 6; GraphPad, San Diego, CA).

\section{Results}

\section{Expression of $\mathrm{P}_{2} \mathrm{Y}_{2}$ receptor $\mathrm{mRNAs}$ during CADSC endothelial differentiation}

Quantitative PCR experiments were performed to evaluate $\mathrm{P}_{2} \mathrm{Y}_{2}$ and $\mathrm{P} 2 \mathrm{Y}_{4}$ UTP receptors expression in cADSC after 7 days in an endothelial differentiation medium. Both receptor expressions were normalized to the housekeeping gene RPL32 expression (Fig. 1A). We have clearly detected a strong expression of the $\mathrm{P} 2 \mathrm{Y}_{2}$ receptor compared to a weak expression of $\mathrm{P}_{2} \mathrm{Y}_{4}$ receptor in cADSC predifferentiated cultures (Fig. 1A). We have then followed the $\mathrm{P}_{2} \mathrm{Y}_{2}$ receptor expression during cADSC endothelial differentiation during 21 days after plating. $\mathrm{P} 2 \mathrm{Y}_{2}$ receptor mRNA expression data displayed a decrease along the endothelial differentiation process (Fig. 1B).

\section{Effect of UTP stimulation of CADSC on their capacity to form vascular network in vitro}

To evaluate the potential effect of UTP, a ligand of the $\mathrm{P} 2 \mathrm{Y}_{2}$ receptor, on the angiogenic properties of CADSC, we have performed in vitro Matrigel angiogenesis assay. cADSC were seeded at a density of $2 \times 10^{4} / 45 \mu \mathrm{L}$ in EGM2 medium after 7 days of endothelial pre-differentiation and a daily stimulation of UTP $100 \mu \mathrm{M}$. Capillary-like tubular structures, visualized using a phase-contrast inverted microscope, were recorded over the course of $20 \mathrm{~h}$, with the automated software Wimasis (Fig. 1C). The data were normalized to untreated CADSC and demonstrated a significant positive effect of UTP treatment on the angiogenic measurements comprising total tube length, mean loop area, and the total number of loop in each endothelial network (Fig. 1D). We have also investigated the potential use of MSCs from abdominal adipose tissue. But using the same endothelial differentiation protocol, it was not possible to differentiate these MSCs into endothelial cells and to generate an endothelial network into Matrigel (data not shown).

\section{UTP target genes in endothelial predifferentiated CADSC}

RNA-sequencing experiments were assessed on cADSC culture after 7 days in endothelial differentiation medium, unstimulated or with a daily UTP $100-\mu \mathrm{M}$ stimulation. MAplot representation provides an overview of regulated-gene distribution within a two-sample comparison. The $y$-values represent the log of the ratio of level counts for each gene between two samples, while the $x$-values are the average level counts for each gene across the two samples, a dot representing one gene (Fig. 2A). Among UTP-regulated genes, we have selected and confirmed by qPCR eleven genes presenting key roles in physiological mechanisms such as proliferation, healing, cardiac tissue repair, inflammation, or angiogenesis (Fig. 2B). Our qPCR experiments confirmed the regulation of the following UTP target genes in predifferentiated cADSC: CXCL10 chemokine, nerve growth factor IB-like receptor $\mathrm{Nr} 4 \mathrm{a} 1$, tenascin $\mathrm{C}$ (Tnc), hyaluronan synthase 1 (Has1), bone morphogenetic protein 2 (Bmp2), epiregulin (Ereg), endothelial cell-specific molecule 1 (Esm1), integrin beta 3 (Itgb3), forkhead box protein $\mathrm{C} 2$ (Foxc2), arginase 1 (Arg1), and glycoprotein CD44 (Fig. 2B). The hyaluronan synthase 1 and epiregulin, two target genes mainly involved respectively in endothelial 

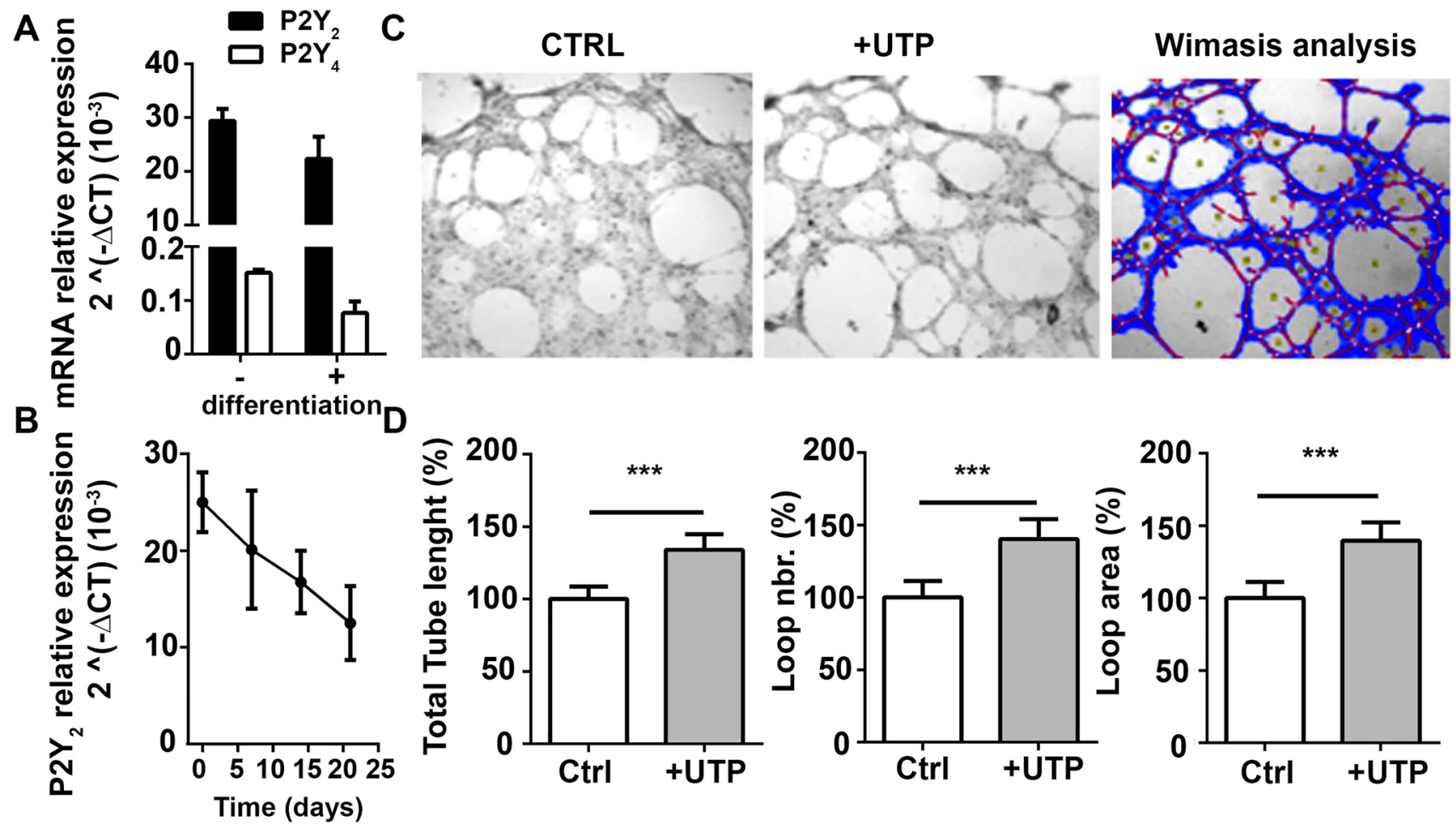

Fig. 1 UTP effect on cADSC endothelial differentiation and vascular network formation. A qPCR analysis showed mRNA level of P2 $\mathrm{Y}_{2}$ and $\mathrm{P}_{2} \mathrm{Y}_{4}$ receptors in cADSC after 7 days in endothelial differentiation medium. B mRNA level of $\mathrm{P}_{2} \mathrm{Y}_{2}$ receptor was determined during differentiation of cADSC into endothelial cells. Values represent mean \pm SEM of mRNA levels measured in 7 (panel A) and 2 (panel B) independent experiments, respectively. C Capillary-like tubular structures of cADSC isolated from wild-type mice, plated for 7 days in endothelial differentiation medium, unstimulated (CTRL), or daily

migration [23] and smooth muscle cell proliferation for tubular formation [24], were quantified by ELISA in the supernatants of predifferentiated cADSC. We observed the UTP positive effect on the release of both hyaluronan synthase 1 (Fig. 2C) and epiregulin (Fig. 2D).

\section{Tracking intramyocardial transplanted UTP-treated predifferentiated CADSC}

The study of UTP effect on proangiogenic properties of endothelial predifferentiated cADSC in post-ischemic revascularization was investigated by performing mouse LAD ligation experiments followed by cADSC injections. EdU tracing was used for in vivo tracking method of transplanted cADSC $2 \mathrm{~h}$ and 4 days after injection (Fig. 3A). As expected, only a small proportion of EdU-positive cADSCs compared to the number of injected cells $\left(3.10^{5}\right.$ cells/ ischemic heart) was detectable in the ischemic tissue after 4 days. Interestingly, we observed a significant increase in the number of EdU-positive engrafted predifferentiated cADSC pretreated with UTP $(52.8 \pm 10.1 \%$, mean \pm SEM; stimulated with UTP $(100 \mu \mathrm{M})$, and then placed on matrigel for $20 \mathrm{~h}$. Tubes and nets were automatically selected and marked in blue using Wimasis Image Analysis System. Blue, the tubular structure. Red, tubes. White, branching points. D Quantitative analysis of tube formation capacity of cADSC was based on three measurements; the relative total tube length, the relative loop number and the relative loop area. Values represent mean \pm SEM using 7 independent experiments. $* * * p<0.001$

$N=4)$ compared to untreated predifferentiated cADSC (Fig. 3B), 4 days after their transplantation. These engrafted predifferentiated cADSC were not positive for Isolectin B4 (IB-4) marker suggesting they do not finalize in vivo their differentiation into mature endothelial cells 4 days after their injection (Fig. 3C).

\section{In vivo proangiogenic effect of UTP-treated predifferentiated CADSC in ischemic heart}

After the setup of UTP-treated cADSC injections in ischemic mouse hearts, we investigated their potential action on cardiac post-infarct revascularization. cADSC were isolated from wild-type mice or $\mathrm{P} 2 \mathrm{Y}_{2}$ receptor $\mathrm{KO}$ mice and treated or not with UTP $100 \mu \mathrm{M}$, daily during a 7-day endothelial pre-differentiation. These untreated or UTP-treated cADSC or control PBS were injected in the peri-infarct border zone, 3 days after LAD ligation. Immunohistochemistry experiments using IB- 4 and $\alpha$-SMA stainings were performed on heart cryosections to quantify respectively capillary and arteriole density in infarct border zone at different levels 
A
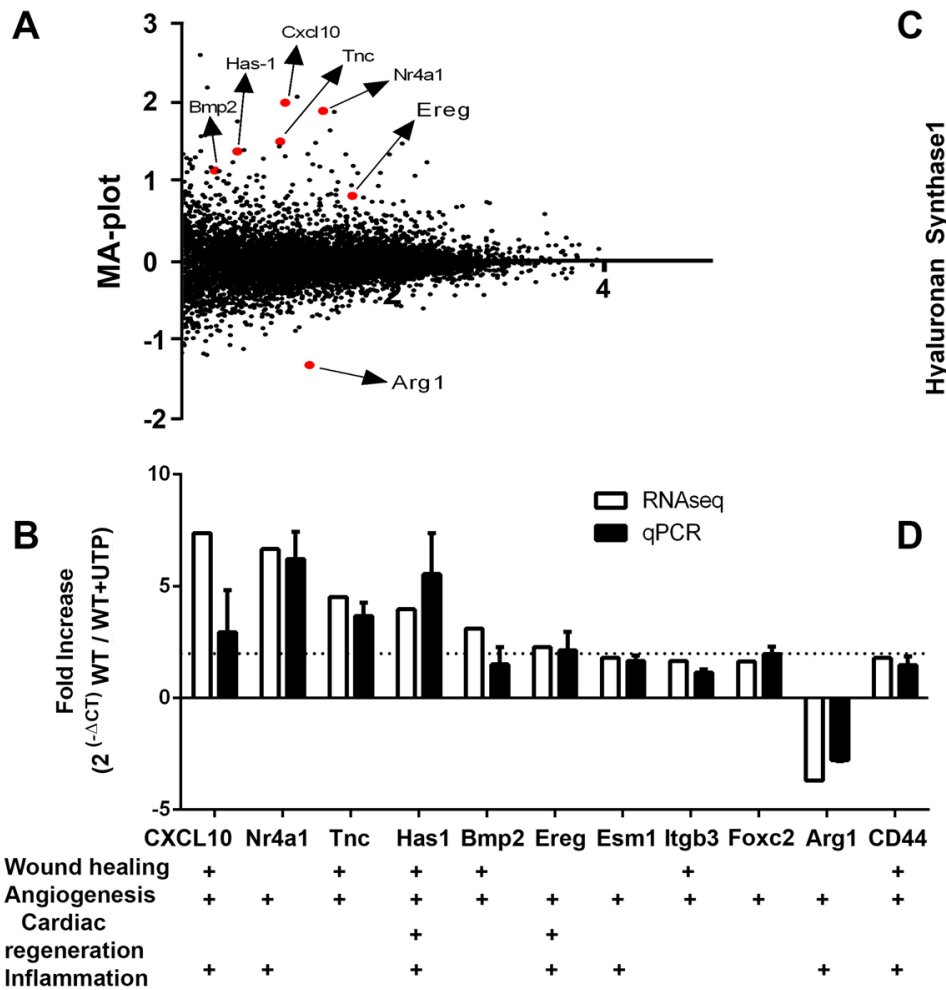

C

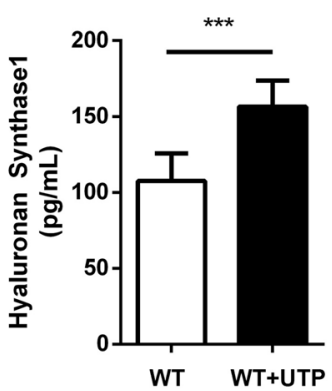

Fig. 2 UTP target genes in predifferentiated cADSC. RNA-sequencing experiments were performed on RNAs from two independent cultures of cADSC after 7 days in the endothelial differentiation medium and daily stimulated with UTP $(100 \mu \mathrm{M})$. A MA-plot shows the average expression on the $\mathrm{X}$-axis and the log fold change on the y-axis of the two groups. B qPCR analysis and confirmation of selected target genes regulated by UTP. mRNA level measurements were performed in four independent experiments for all genes. C Hyaluronan synthase-1 and epiregulin levels were measured in supernatants of cADSC cultures after 7 days in the endothelial dif- ferentiation medium with or without daily stimulation with UTP $100 \mu \mathrm{M}$. ELISA data were obtained in four independent experiments. Nr4a1 nerve growth factor IB, Tnc tenascin C, Has 1 hyaluronan synthase 1, BMP2 bone morphogenetic protein 2, Ereg epiregulin, Esm1 endothelial cell-specific molecule 1, Itgb3 Integrin beta 3 , Foxc2 forkhead box protein $\mathrm{C} 2$, Arg1 arginase 1. Values represent mean \pm SEM. $* p<0.05$ and $* * * p<0.001$. MA plot; $\mathrm{X}=\ln \left(\sqrt{ }\left(\times 1^{*} \times 2\right)\right), \mathrm{Y}=\log _{2}(\times 1 / \times 2)$. ELISA, enzyme-linked immunosorbent assay

performed to quantify cardiac fibrosis in these ischemic transplanted hearts. Thirty days after cADSC injection, we observed a significant reduction of cardiac fibrosis in mice transplanted with UTP-treated cADSC compared to mice transplanted with untreated cADSC (decrease of $32 \pm 12 \%$; mean \pm SEM; $*: p<0.05)$.

\section{Discussion}

Proangiogenic strategies for myocardial repair in cardiovascular diseases still raise many investigations on therapeutic applications. Early reperfusion by restoration of blood flow and oxygen supply is the only way to rescue the ischemic myocardium by restricting the infarction size and improving clinical outcomes [1]. The current conventional therapeutic treatments for myocardial infarction, despite improving quality of life, have limited success in preventing the progression of left ventricular remodeling, resulting in a partial 

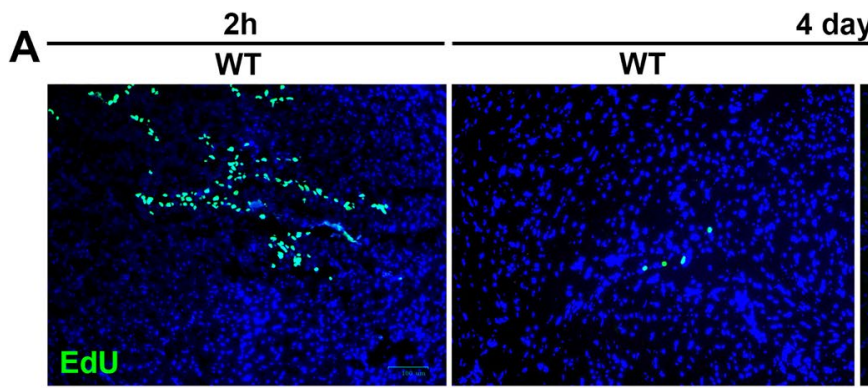

4 days
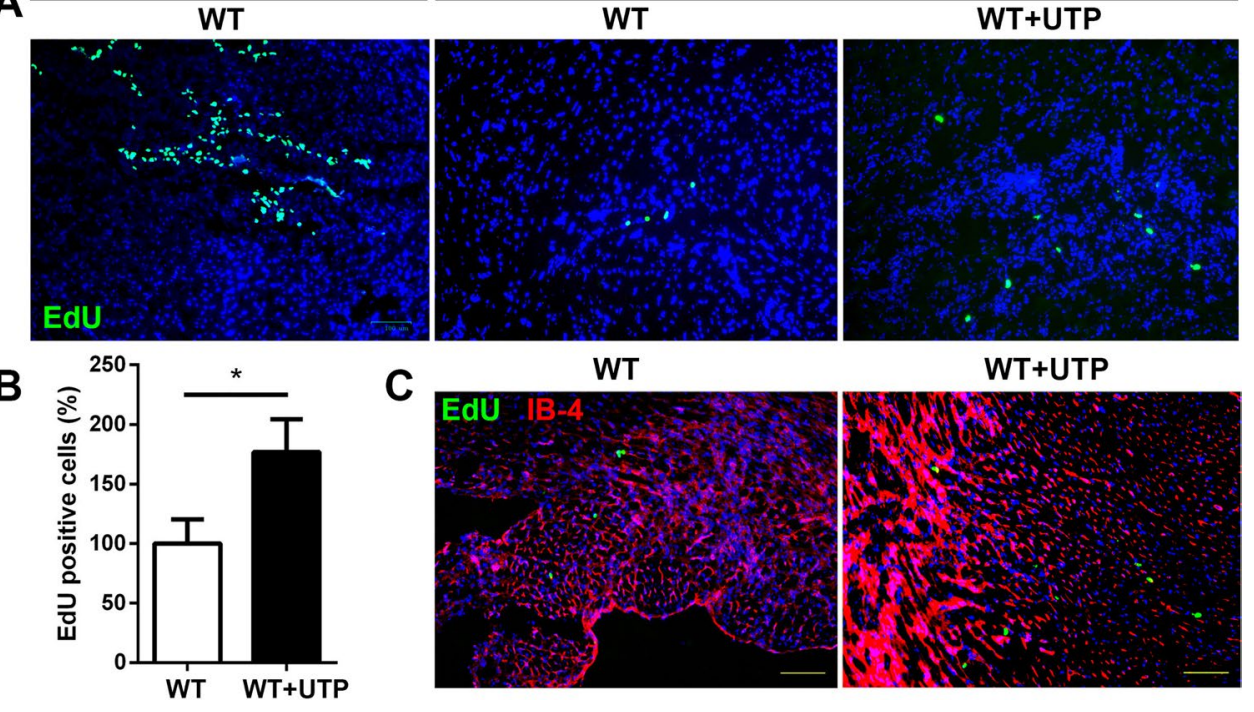

WT+UTP

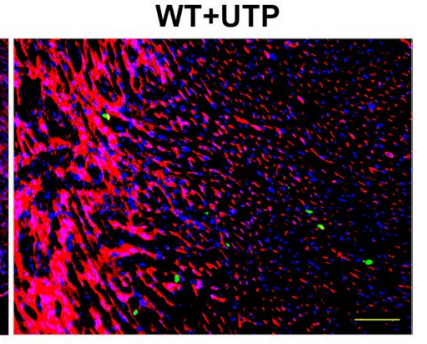

Fig. 3 Tracking of intramyocardial transplanted UTP-treated predifferentiated cADSC. cADSC treated or not with UTP were labeled with EdU at $10 \mu \mathrm{M}$ and injected in 3 days post-MI hearts. A Representative images showing the number of EdU-positive endothelial predifferentiated cADSC in the peri-infarct border zone, $2 \mathrm{~h}$ or 4 days after their injection. EdU (green), Hoechst (blue); WT $=$ control $\mathrm{cADSC}, \mathrm{WT}+\mathrm{UTP}=\mathrm{cADSC}$ treated daily with UTP $100 \mu \mathrm{M}$ during endothelial pre-differentiation. B Quantification of EdU-positive cells in the peri-infarct border zone, 4 days after injection of predifferentiated cADSC untreated or treated with UTP. The percentage

restoration of heart function [25]. Adipose-derived stem cell-based therapy holds promise for tissue regeneration and revascularization by stimulating the secretion of various cytokines responsible for proangiogenic and antiapoptotic effects [7]. The feasibility and efficacy of ADSC have been already tested in clinical trials [26]. Nevertheless, there are multiple challenges to solve accurately before ADSC-based cell therapy becoming a routine clinical procedure. Over the last few years, specific attention has been observed to elucidate and regulate the proangiogenic effects of injected ADSC for regeneration and revascularization purposes.

In the present study, we have investigated the potential action of P2Y nucleotide receptors in the endothelial commitment of ADSC from cardiac fat and in their angiogenic properties.

The cardiac adipose tissue can have a regulatory role in cardioprotection due to its proximity to the myocardium and coronary arteries. Nevertheless, its small size limits the generation of stem cells and the setup of a potential human cell therapy. We have investigated the potential use of MSCs from abdominal adipose tissue. The major problem with these MSCs was the difficulty to differentiate them into endothelial cells. Only MSCs from cardiac fat were able to differentiate into functional endothelial cells and generate an endothelial network into Matrigel. Moreover, we have represents the number of EdU-positive cells found in 20-25 fields per heart, harvested from 4 mice per condition. C Representative images showing the number of EdU-positive cADSC in the periinfarct border zone, 4 days after their injection. Isolectin B4 (IB-4) (purple), EdU (green), Hoechst (blue); WT=untreated cADSC, $\mathrm{WT}+\mathrm{UTP}=\mathrm{cADSC}$ treated daily with UTP $100 \mu \mathrm{M}$ during endothelial pre-differentiation. Pictures were obtained using Zeiss microscope at $\times 100$ magnification, scale bar represents $50 \mu \mathrm{m}$. Data are expressed as mean \pm SEM and are representative of an experiment of two. ${ }^{*} p<0.05$

previously shown that $\mathrm{P}_{2} \mathrm{Y}_{2}$ receptor was detected in SC from abdominal adipose tissue but displayed no functional role in adipogenesis contrary to its role in cardiac adipose tissue [20]. All these elements have determined our choice to use SC from cardiac adipose tissue in our experiments but of course, we do not exclude that MSCs from abdominal adipose tissue could be an option in the context of a human therapy based on ADSC injection.

To study the effect of UTP on vascular network formation from predifferentiated cADSC, we have performed in vitro angiogenic Matrigel assays. Capillary-like tubular structure analyses revealed a positive effect of UTP treatment in specific measurements screening the angiogenic activity of endothelial predifferentiated cADSC. Previous studies describe the role of extracellular nucleotides as potential pivotal regulators in early mesenchymal stem cell commitment, specifically in osteogenic and adipogenic differentiation of human dental follicle cells and adipose-derived stem cells from liposuction aspirate [18]. According to our data, it appears that UTP has an interesting contribution in the regulation of cADSC endothelial differentiation and their angiogenic properties.

Our RNA-sequencing experiments identified UTP target genes potentially linked to cADSC endothelial differentiation and angiogenic properties. We identified genes involved 
A

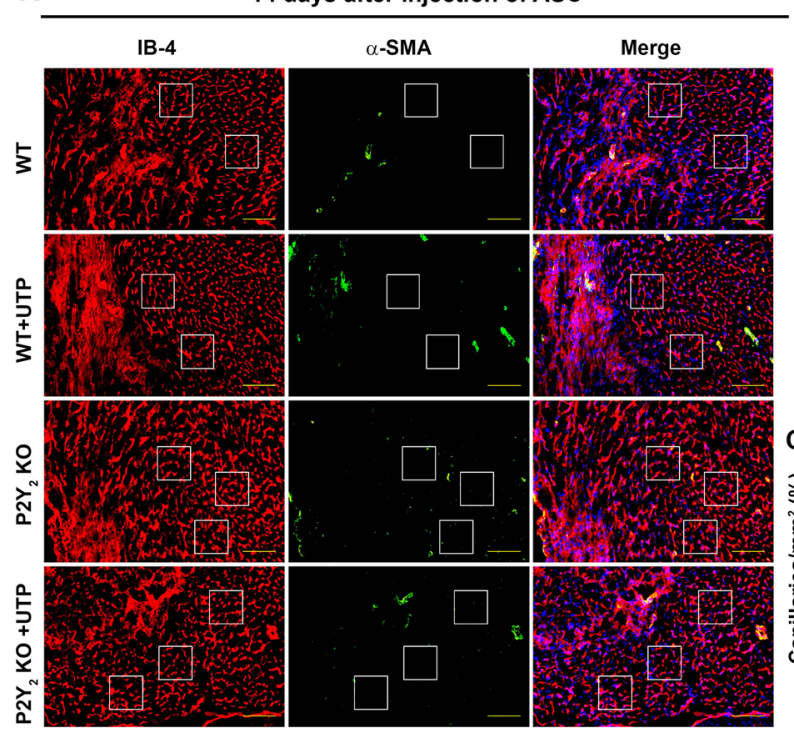

B

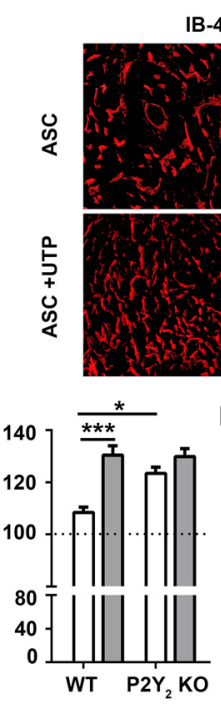

30 days after injection of ASC
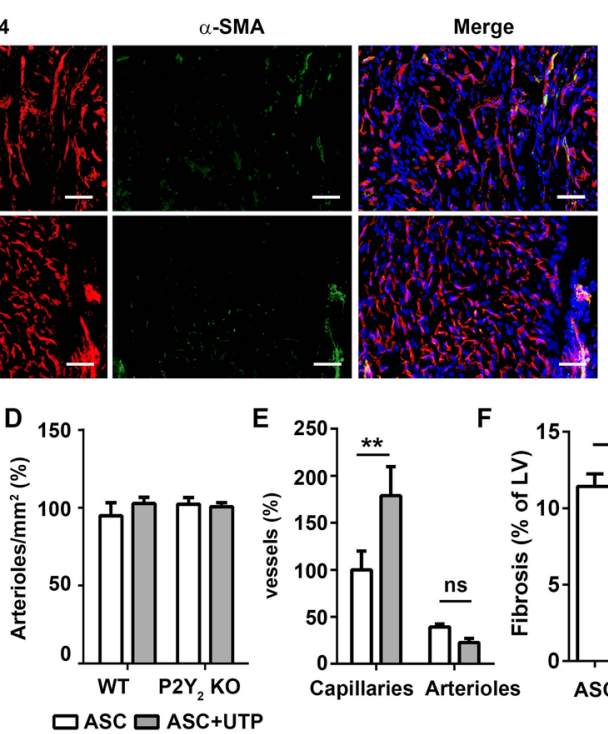

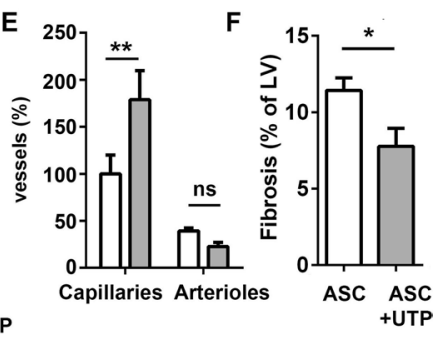

Fig. 4 In vivo action of injection of UTP-treated WT or $\mathrm{P}_{2} \mathrm{Y}_{2}$ receptor $\mathrm{KO}$ cADSC in MI hearts. A Immunofluorescence images of Isolectin B4 (purple), $\alpha$-smooth muscle actin (green), and Hoechst (blue) in the peri-infarct border zone, 14 days after injection of WT or $\mathrm{P} 2 \mathrm{Y}_{2}$ receptor KO cADSC untreated or treated with UTP (daily stimulation with $100 \mu \mathrm{M}$ UTP during endothelial pre-differentiation). B Immunofluorescence images of Isolectin B4 (purple), $\alpha$-smooth muscle actin (green), and Hoechst (blue) in the peri-infarct border zone, 30 days after injection of untreated or UTP-treated WT or $\mathrm{P}_{2} \mathrm{Y}_{2}$ receptor KO predifferentiated cADSC. C Representative counting surface/field $\left(0.1 \mathrm{~mm}^{2}\right)$ of capillary density quantification, based on IB4-staining, 14 days after injection of untreated or UTP-treated WT or $\mathrm{P}_{2} \mathrm{Y}_{2}$ receptor KO cADSC in WT ischemic hearts. D Representative counting surface/field $\left(0.1 \mathrm{~mm}^{2}\right)$ of arteriole density $(\alpha-\mathrm{SMA}+/$ IB4+) quantification, 14 days after injection of untreated or UTPtreated WT or $\mathrm{P} 2 \mathrm{Y}_{2}$ receptor $\mathrm{KO}$ cADSC in WT ischemic hearts in 3 independent experiments. E Histograms showing quantitative data of capillary (IB4+) and arteriole density $(\alpha-\mathrm{SMA}+/ \mathrm{IB} 4+)$ in the peri-infarcted ventricle, 30 days after injection of untreated or UTPtreated WT cADSC in WT ischemic hearts in two independent experiments. F Histograms showing quantitative data of fibrosis (Masson trichrome staining) normalized to left ventricle area in WT ischemic hearts 30 days after injection of untreated or UTP-treated WT cADSC. Capillary density was calculated in around 60 fields (at $\times 100$ magnification) per ischemic heart, and the final data expressed in percentage are normalized to the PBS injection condition. Arteriole density was calculated in around 20 fields per ischemic heart and normalized to the PBS injection condition. Fibrosis was quantified as the relative surface of the blue staining on five cryosections per ischemic heart using ImageJ software and normalized to the left ventricle area. Scale bar represents $50 \mu \mathrm{m}$. Values are presented as mean \pm SEM ( $n=3-6$ mice per group). $* p<0.05,{ }^{*} p<<0.01, * * * p<0.001$, and $\mathrm{ns}$, not significant

cells in ischemic tissue, 4 days after injection. We therefore evaluated the revascularization of the ischemic heart 14 and 30 days after intramyocardial injection of predifferentiated cADSC. Capillary density quantification in the ischemic border zone allowed the evaluation of the effect of injection of UTP-treated cADSC on post-ischemic cardiac revascularization. Interestingly, 14 days and 30 days after injection of cADSC, higher capillary density was found around the necrotic zone using cADSC treated with UTP during endothelial predifferentiation. We used UTP instead of ATP to prestimulate ADSC to avoid ADP or adenosine receptor activation due to ATP degradation. Our observed in vivo effect of UTP-treated cardiac ADSCs on cardiac revascularization was lost using UTP-treated $\mathrm{P}_{2} \mathrm{Y}_{2} \mathrm{KO}$ ADSCs. The rapid elimination of endothelial-differentiated ADSC supposes that they do not integrate into newly formed capillaries. Nevertheless, the presence of ADSC in the first hours after injection could initiate cardiac revascularization 
through a proangiogenic paracrine signal such as released angiogenic factors identified by RNA-sequencing.

Many inflammatory signals are released during ischemia including extracellular nucleotides and can have multiple actions on injected ADSC. No significant effect of injection of untreated ADSC vs PBS injection was observed on cardiac revascularization. The positive effect of UTP treatment of injected cADSC on capillary density was not observed using cADSC isolated from $\mathrm{P}_{2} \mathrm{Y}_{2}$ receptor $\mathrm{KO}$ mice. Altogether, these in vivo data indicate that the activation of $\mathrm{P} 2 \mathrm{Y}_{2}$ receptor exerts a prevalent action on the revascularization capacities of injected cADSC. The reduction of cardiac scar size 30 days after UTP-treated cADSC injection in ischemic mice supports their cardioprotective action in the ischemic heart.

Before injection, ADSCs were daily stimulated with 100$\mu \mathrm{M}$ UTP during endothelial pre-differentiation. It is important to note that supernatants of UTP-treated ADSC were removed to count cells before injection in ischemic heart. The effects on cardiac revascularization are thus not due to remaining UTP, its degradation products, or any secreted proteins in the ADSC supernatants, but more likely to the preconditioning of treated ADSC. As reflected by our RNA-sequencing experiments, the presence of UTP in their endothelial differentiation medium affects their gene profile and their capacity to exert paracrine actions to participate in revascularization. The release of extracellular nucleotides in the injured microenvironment and ectonucleotidase expression on cardiac cells can then also influence the action of injected ADSC.

A cardioprotective effect of UTP treatment has already been described in ischemic mice and was lost in $\mathrm{P}_{2} \mathrm{Y}_{2} \mathrm{KO}$ mice [27]. UTP reduces infarct size and improves mice heart function after myocardial infarct via the $\mathrm{P} 2 \mathrm{Y}_{2}$ receptor [27]. Pyrophosphate, a degradation product of UTP, was able to protect cardiomyocytes from hypoxic damage but displays no cardioprotective effect in vivo [27]. A therapeutic approach using UTP injection is difficult to propose due to its rapid degradation and the ubiquitous expression of $\mathrm{P}_{2} \mathrm{Y}_{2}$ with possible multiple side effects on immune cells and other cardiac cell types than cardiomyocytes. The present study aims to propose a cardioprotective treatment focused on revascularization initiated by injection of endothelial predifferentiated ADSC prestimulated with UTP. ATP is likely to have similar effects than UTP on $\mathrm{P}_{2} \mathrm{Y}_{2}$ activation expressed on ADSC due to their equipotency but ATP was not considered as a stimulating agent in our in vivo experiments to avoid side effects of its degradation products that could occur through ADP or adenosine receptor activation.

The combination of in vivo action of UTP-treated transplanted cADSC in ischemic heart and in vitro data of endothelial network formation in Matrigel is very promising. The present study defines UTP ligand and $\mathrm{P}_{2} \mathrm{Y}_{2}$ receptor as potential regulators of the therapeutic use of cADSC against cardiac ischemia.

It is noteworthy that the modest engraftment of intramyocardial injected stem cells, previously noted in other studies [28], strengthened the importance of injected-stem cell paracrine effects. Stem cell preconditioning strategies like hypoxia, growth factors, and conditioned medium are well known to improve cell functions such as their paracrine ability to release proangiogenic factors [29]. A previous study has proved that endothelial differentiation of MSC markedly proved to diminish their ability to differentiate into adipocytes and other cell types [30]. In the context of cell-based therapies, this could reduce potential problems because of the differentiation of MSC into undesired cell types. The combination of endothelial predifferentiation and UTP treatment of CADSC before their injection might provide a new approach to enhance angiogenic capacities/ paracrine effects in post-ischemic revascularization stem cell-based therapy.

The angiogenic effect of UTP-treated cADSC in postischemic heart could be due to an increase in their ability to secrete proangiogenic factors, as identified by our RNA-sequencing experiments. It is promising to observe that UTP treatment of cADSC gave rise to a proangiogenic effect on capillary density on the peri-infarcted zone and reduction of fibrosis area in the ischemic heart, 30 days after their injection. Providing a better understanding of the angiogenic phenomenon observed in our in vivo study may however represent a crucial step from a clinical perspective.

Our data provide new important insights into the optimization of cell therapy for cardiac repair and post-ischemic revascularization, based on purinergic receptor activation/ regulation.

This study defines $\mathrm{P}_{2} \mathrm{Y}_{2}$ receptor as a pivotal actor to regulate ADSC differentiation and enhance their proangiogenic capacities might be a promising approach to significantly enlarge stem cell therapy benefits.

Funding This work was supported by the Research Project and Research Credit of the Fonds National de la Recherche Scientifique of Belgium (J.0060.18 CDR grant), by an ATIMI (Attract Brains for Brussels, Belgium) grants of Innoviris Brussels (2016 BB2B6 ATT RACT and 2019-BFB-106 ATIMI grants), by the Fund Lokumo, King Baudouin Foundation, Belgium (2016-B7131100-204941 and 2017B7131100-207336 grants), by Action de Recherche Concertée of the Communauté Française de Belgique, by an Interuniversity Attraction Pole grant from the Politique Scientifique Fédérale (IAP-P6/30), Prime Minister's Office, Federal Service for Science, Technology and Culture, by the Fonds d'Encouragement à la Recherche (F.E.R.), by the Fonds Emile DEFAY, and by the LifeSciHealth programme of the European Community (grant LSHB-2003-503337). M. Vanorlé was supported by the F.RI.A., Fonds National de la Recherche Scientifique / FRIA, Belgium. A. Lemaire and L. di Pietrantonio are supported by ULB, Belgium. M. Horckmans is supported by an ATIMI (Attract 
Brains for Brussels) grant of Innoviris Brussels. D. Communi is Senior Research Associate of the Fonds National de la Recherche Scientifique (F.N.R.S.)

Data availability The datasets generated during and/or analyzed during the current study are available from the corresponding author on reasonable request.

\section{Declarations}

Ethical approval All animal work has been conducted in accordance with the European Community guidelines for the care and use of laboratory animals and approved by the ethics committee of the Free University of Brussels (current approved protocols 2019/659 N and 2019/714 N).

Conflicts of interest Marion Vanorlé declares that she has no conflict of interest.

Anne Lemaire declares that she has no conflict of interest. Larissa di Pietrantonio declares that she has no conflict of interest. Michael Horckmans declares that he has no conflict of interest Didier Communi declares that he has no conflict of interest. Disclaimer The funders had no role in study design, data collection, and analysis, decision to publish, or preparation of the manuscript.

\section{References}

1. Lévy B, Smadja D, Silvestre J-S (2013) Ischémie tissulaire et régénération vasculaire: Mécanismes moléculaires et cellulaires, perspectives thérapeutiques. John Libbey Eurotext

2. Zhao L, Johnson T, Liu D (2017) Therapeutic angiogenesis of adipose-derived stem cells for ischemic diseases. Stem Cell Res Ther 8(1): 125

3. Yang D, Wang W, Li L, Peng Y, Chen P, Huang H, Guo Y, Xia $\mathrm{X}$, Wang $\mathrm{Y}$, Wang $\mathrm{H}$, Wang WE, Zeng $\mathrm{C}$. The relative contribution of paracine effect versus direct differentiation on adiposederived stem cell transplantation mediated cardiac repair. PloS One. 2013;8(3): 559020.

4. Chen L, Qin F, Ge M, Shu Q, Xu J (2014) Application of adipose-derived stem cells in heart disease. J Cardiovasc Transl Res 7:651-663

5. Cai L, Johnstone BH, Cook TG, Tan J, Fishbein MC, Chen P-S, March KL (2009) IFATS collection: human adipose tissue-derived stem cells induce angiogenesis and nerve sprouting following myocardial infarction, in conjunction with potent preservation of cardiac function. Stem Cells 27:230-237

6. Schenke-Layland K, Strem BM, Jordan MC, DeEmedio MT, Hedrick MH, Roos KP, Fraser JK, MacLellan WR (2009) Adipose tissue-derived cells improve cardiac function following myocardial infarction. J Surg Res 153:217-223

7. Gnecchi M, Zhang Z, Ni A, Dzau VJ (2008) Paracrine mechanisms in adult stem cell signaling and therapy. Circ Res 103:1204-1219

8. Stubbs SL, Hsiao ST-F, Peshavariya HM, Lim SY, Dusting GJ, Dilley RJ (2012) Hypoxic preconditioning enhances survival of human adipose-derived stem cells and conditions endothelial cells in vitro. Stem Cells Dev 21:1887-1896

9. Zhu P, Liu J, Shi J, Zhou Q, Liu J, Zhang X, Du Z, Liu Q, Guo Y (2015) Melatonin protects ADSCs from ROS and enhances their therapeutic potency in a rat model of myocardial infarction. $\mathrm{J}$ Cell Mol Med 19:2232-2243
10. Nagata H, Ii M, Kohbayashi E, Hoshiga M, Hanafusa T, Asahi M (2016) Cardiac adipose-derived stem cells exhibit high differentiation potential to cardiovascular cells in C57BL/6 mice. Stem Cells Transl Med 5:141-151

11. Dutta AK, Sabirov RZ, Uramoto H, Okada Y (2004) Role of ATP-conductive anion channel in ATP release from neonatal rat cardiomyocytes in ischaemic or hypoxic conditions: ATPconductive channel in ischaemic cardiomyocytes. J Physiol 559:799-812

12. Lustig KD, Shiau AK, Brake AJ, Julius D (1993) Expression cloning of an ATP receptor from mouse neuroblastoma cells. Proc Natl Acad Sci U S A 90:5113-5117

13. Yitzhaki S, Shneyvays V, Jacobson KA, Shainberg A (2005) Involvement of uracil nucleotides in protection of cardiomyocytes from hypoxic stress. Biochem Pharmacol 69:1215-1223

14. Communi D, Pirotton S, Parmentier M, Boeynaems JM (1995) Cloning and functional expression of a human uridine nucleotide receptor. J Biol Chem 270:30849-30852

15. Horckmans M, Bianchini M, Santovito D, Megens RTA, Springael J-Y, Negri I, Vacca M, Di Eusanio M, Moschetta A, Weber C, Duchene J, Steffens S (2017) Pericardial adipose tissue regulates granulopoiesis, fibrosis and cardiac function after myocardial infarction. Circulation

16. Glaser T, Cappellari AR, Pillat MM, Iser IC, Wink MR, Battastini AMO, Ulrich H (2012) Perspectives of purinergic signaling in stem cell differentiation and tissue regeneration. Purinergic Signal 8:523-537

17. Kaebisch C, Schipper D, Babczyk P, Tobiasch E (2015) The role of purinergic receptors in stem cell differentiation. Comput Struct Biotechnol J 13:75-84

18. Zippel N, Limbach CA, Ratajski N, Urban C, Luparello C, Pansky A, Kassack MU, Tobiasch E (2012) Purinergic receptors influence the differentiation of human mesenchymal stem cells. Stem Cells Dev 21:884-900

19. Lemaire A, Vanorlé M, Horckmans M, di Pietrantonio L, Clouet S, Robaye B et al (2017) Mouse P2Y4 nucleotide receptor is a negative regulator of cardiac adipose-derived stem cell differentiation and cardiac fat formation. Stem Cells Dev 26(5):363-373

20. Negri I, Diaz Villamil E, De Roeck L, Communi D, Horckmans M (2019) P2Y2 nucleotide receptor is a regulator of the formation of cardiac adipose tissue and its fat-associated lymphoid clusters. Stem Cells Dev 29(2):100-109

21. Homolya L, Watt WC, Lazarowski ER, Koller BH, Boucher RC (1999) Nucleotide-regulated calcium signaling in lung fibroblasts and epithelial cells from normal and $\mathrm{P} 2 \mathrm{Y}_{2}$ receptor (-/-) Mice. J Biol Chem 274:26454-26460

22. Laplante M-A, Monassier L, Freund M, Bousquet P, Gachet C (2010) The purinergic P2Y1 receptor supports leptin secretion in adipose tissue. Endocrinology 151:2060-2070

23. Jiang D, Liang J, Noble PW (2007) Hyaluronan in tissue injury and repair. Annu Rev Cell Dev Biol 23:435-461

24. Riese DJ, Cullum RL (2014) Epiregulin: roles in normal physiology and cancer. Semin Cell Dev Biol 28:49-56

25. Kwon Y-W, Yang H-M, Cho H-J (2010) Cell therapy for myocardial infarction. Int J Stem Cells 3:8-15

26. Nguyen PK, Rhee J-W, Wu JC (2016) Adult stem cell therapy and heart failure, 2000 to 2016: a Systematic Review. JAMA Cardiol 1:831

27. Cohen R, Shainberg A, Hochhauser E, Cheporko Y, Tobar A, Birk E, Pinhas L, Leipziger J, Don J, Porat E (2011) UTP reduces infarct size and improves mice heart function after myocardial infarct via P2Y2 receptor. Biochem Pharmacol 82:1126-1133

28. Patel NG, Zhang G (2014) Stacked stem cell sheets enhance cell-matrix interactions. Organogenesis 10:170-176 
29. Baraniak PR, McDevitt TC (2010) Stem cell paracrine actions and tissue regeneration. Regen Med 5:121-143

30. Liu JW, Dunoyer-Geindre S, Serre-Beinier V, Mai G, Lambert J-F, Fish RJ, Pernod G, Buehler L, Bounameaux H, Kruithof EKO (2007) Characterization of endothelial-like cells derived from human mesenchymal stem cells. J Thromb Haemost $5: 826-834$
Publisher's note Springer Nature remains neutral with regard to jurisdictional claims in published maps and institutional affiliations. 